\title{
THE STRUCTURE OF TOPOLOGICAL SEMIGROUPS
}

\author{
A. D. WALLACE
}

The title of this address might incline one to the notion that here is to be found a small number of large theorems. To the contrary, I shall talk about a large number of small theorems. Actually, there does not exist at this time any corpus of information to which the title "structure of topological semigroups" is in any fashion applicable. Whether such a body of theorems will ever exist is a matter for the future and is likely to depend on the use to which it might be put as well as to the tastes of mathematicians who are not yet such.

When the investigation of topological groups began there was at hand a theory of abstract groups and much of a fundamental character in Lie groups was available. Beyond this there existed a great body of geometry even if some of it was in a nebulous state insofar as the then held standards of rigor were concerned.

With topological semigroups the situation is quite contrariwise. Here we are faced with a lack of satisfactory algebraic results. I do not think that there are so many as twenty-five papers each exceeding ten pages which are concerned exclusively with the algebraic aspects.

We are more fortunate than were the pioneers who forayed the frontiers of topological groups in that we have at our disposal a greater wealth of topology. Much that they could not-or at least did not-use is at hand for our use. Furthermore we can rely, at least if for no more than analogy, on their results. The state of both algebraic and set-theoretic topology is a somewhat happier one now than then. Still we are likely to be troubled for awhile for lack of something like Haar measure without which we shall be at a loss for representation theorems. At present there seems to be no line of attack on the representation problem and it is probable that we shall need to rely to a greater extent on geometry and topology than was the case with groups.

1. Introduction. My interest in this field began many years ago when, as a graduate student, I first learned of the beautiful theorem of E. Cartan,

If an n-sphere is a topological group then $n=0,1$ or 3 .

An address delivered before the Annual Meeting of the Society in Baltimore, Maryland, on December 28, 1953, by invitation of the Committee to Select Hour Speakers for Annual and Summer Meetings; received by the editors July 2, 1954. 
No thought is required of a graduate student to turn this into an all-embracing question-

What spaces admit what algebraic structures?

But my training and a few simple examples indicated that more progress was likely to be made if the question were reformulated;

What compact connected Hausdorff spaces admit a continuous associative multiplication with unit?

The question is still a large one. We shall concentrate on other, and shorter, themes and leave the original as a basso ostinato, an insistent reminder not to stray too far, in our development, from the announced problem.

A $m o b$ is a map (=continuous function) $m: S \times S \rightarrow S$ such that

(i) $S$ is a Hausdorff space and

(ii) $m$ is associative. If we write $x y=m(x, y)$ then (ii) becomes the more familiar $(x y) z=x(y z)$.

A word about terminology is in order. One commonly uses such phrases as " $G$ is a compact topological group" when, of course, $G$ is actually a set. Tradition will be followed here and we will use " $S$ is a compact mob" in place of a longer and more correct form.

A clan is a compact connected mob with a (two-sided) unit. A schematic theorem of the sort that interests me is as follows:

THEOREM. $H_{1}: S$ is a clan

$\mathrm{H}_{2}$ : Topological

C: Algebraic.

Let us say that a space is indecomposable if it is not the union of two closed connected proper subsets. We commonly think of indecomposable spaces as being monstrous things created by set-theoretic topologists for some evil (but purely mathematical) purpose.

THEOREM. $H_{1}: S$ is a clan

$$
\begin{aligned}
& H_{2}: S \text { is indecomposable } \\
& C: S \text { is a group. }
\end{aligned}
$$

A manifold is a locally Euclidean space. Surely manifolds and indecomposable spaces are antipodal points in the sphere of topology. But we modulate from the tonic minor of the pathological (by way of algebraic topology) to the dominant major of the commonplace to obtain this result,

TheOREM. $H_{1}: S$ is a clan $\mathrm{H}_{2}: \mathrm{S}$ is a manifold

$C: S$ is a group. 
Of course not all theorems of interest fall into the scheme we indicated. Let $R^{n}$ be $n$-dimensional Euclidean space. Denote the (settheoretic) boundary of the set $A$ by $F(A)$. Now if $u$ is the unit of the clan $S$ let $H(u)$ be the set of all elements of $S$ with (two-sided) inverses relative to $u$. As we shall see later $H(u)$ is a compact group.

THEOREM. $H_{1}: S$ is a clan

$\mathrm{H}_{2}: S$ is topologically contained in $R^{n}, n \geqq 2$.

$C: H(u) \subset F(S)$.

With this introduction we turn to the pedestrian task of indicating in more detail what has been accomplished thus far in mobs. We shall make a definite effort to show that there are few domains of topology into which mob-theory has not penetrated. We shall not mention the work of Eckmann, Hopf, Leray, Borel and others discussed by Samelson in his invited address [25]. The applications to analysis were discussed by Hille in his Colloquium Lectures [13].

A word about notation and terminology. In general, topological meanings will have precedence of algebraic ones. We use $A^{*}$ for the topological closure of $A$ so that " $A$ is closed" means $A=A$ * and not $A^{2} \subset A$. We denote the null set by $\square, 0$ and $\square$ being homeomorphic. The symbol $A \backslash B$ is used rather than $A-B$ for the complement of $B$ in $A$. "Compact" will be used for "bicompact." A space is connected if it is not the union of two nonvoid disjoint closed sets.

2. Maximal subgroups. It is natural to examine first those substructures of the mob $S$ (and in all that follows $S$ will be a mob) with which we are most familiar. A subgroup of $S$ is a nonvoid set $A$ satisfying $x A=A=A x$ for all $x \in A$. With the aid of the Hausdorff Maximality Principle (or Zorn's Lemma) one readily proves [32]:

THEOREM 2.1. Each subgroup of $S$ is contained in a maximal such and no two of these intersect.

It may happen that $S$ contains no subgroups. But when $S$ is compact there is at least one. Now a subgroup is a self-contained object, and, in order to bring into play all of $S$, we say that a set $L \subset S$ is a left ideal if $L \neq \square$ and if $S L \subset L$. Here, $A B=\{x y \mid x \in A$ and $y \in B\}$ or $A B=m(A \times B)$. Similarly one defines right ideal and (two-sided) ideal. We shall denote by $K$ the (unique if it exists) minimal ideal of $S$ and by $E$ all those $e \in S$ such that $e^{2}=e$. Recall that if $S$ has a (twosided) unit it is designated by $u$. If $e$ is an idempotent (i.e., $e \in E$ ) let $H(e)$ be the maximal subgroup of $S$ containing $e$.

THEOREM 2.2. If $S$ has a minimal left and a minimal right ideal then 
$S$ has a minimal ideal $K$ and

$$
K=U\{H(e) \mid e \in E \cap K\} .
$$

Any pair $H\left(e_{1}\right), H\left(e_{2}\right)$ of subgroups with $e_{1}, e_{2} \in E \cap K$ are topologically isomorphic.

This result goes back to Suschkewitsch [27], Rees [24], and is due essentially to Clifford [3] with these hypotheses. It is useful to note that $H(e)=e S e$ if and only if $e \in K$. Recall that a space $X$ has the fixed point property if each map $f: X \rightarrow X$ provides an $a \in X$ such that $f(a)=a$. When the hypotheses of Theorem 2.2 subsist and if $S$ has the fixed point property, then $K \subset E$. It is interesting to know what properties of $S$ are inherited by $K$. Thus if $S$ is an absolute retract (absolute neighborhood retract) in the sense of Borsuk, is $K$ also one such? This is true if, for example, $S$ is abelian or indeed if $S$ contains a (nonvoid) normal subset because then $K=e S e$ and the function $x \rightarrow$ exe retracts $S$ onto $K$, see Clifford-Miller [5] and Koch [15]. We shall see later that, if $S$ is a clan, then the cohomology structure of $K$ is exactly that of $S$.

The maximal subgroups of $S$ seem rather mysterious beings. Here is a result reformulated from Green [12].

THEOREM 2.3. Let, for $x \in S$,

$$
T(x)=x S \cap S x \cap\{y \mid x \in y S \cap S y\} .
$$

Then $T(e)=H(e)$ if $e \in E$ and $T(x)$ is a maximal subgroup if and only if $T(x)$ is a submob.

Theorem 2.4. Let $S$ be compact and let

$$
H=\bigcup\{H(e) \mid e \in E\} .
$$

Then $H$ is closed. If $x \in H$ let $y(x)$ be the unit of the unique maximal subgroup containing $x$ and let $\theta(x)$ be the inverse of $x$ in this group. Thus

$$
\begin{aligned}
x y(x) & =x=y(x) x, \\
x \theta(x) & =y(x)=\theta(x) x, \\
y(y(x)) & =y(x), \quad \theta(\theta(x))=x .
\end{aligned}
$$

Then $y: H \rightarrow E$ is a retraction and $\theta: H \rightarrow H$ is an involutorial homeomorphism.

This result (see [32]) shows that $H$ has a "continuous unit" and a "continuous inverse." It is a corollary that when $S$ is a compact algebraic group it is also a topological group. It does not yet seem to 
be known if "locally compact" can replace "compact" in the last sentence. A useful algebraic analysis of $H$ can be found in Clifford [2] under the condition that $H H \subset H$.

There is a sort of dual to Theorem 2.4 (see $[1 ; 9 ; 18$ and 34$]$ ). Let

$$
P(S)=\{x \mid x S=S\}, \quad Q(S)=\{x \mid S x=S\} .
$$

Theorem 2.5. Let $S$ be compact and let $P(S) \neq \square$. Then

$$
P(S)=U\{H(e) \mid e \in E \cap P(S)\}
$$

and each pair of the groups $H\left(e_{1}\right), H\left(e_{2}\right)$ are topologically isomorphic. Indeed, if $e_{0} \in E \cap P(S)$, there is a topological isomorphism

$$
P(S)=H\left(e_{0}\right) \times(E \cap P(S)) .
$$

Moreover $E \cap P(S)$ is the set of left units of $S$. Finally $P(S) \neq \square \neq Q(S)$ if and only if $S$ has a unit and then $P(S)=H(u)=Q(S)$.

In a sense Theorem 2.5 is "best possible." For if $G$ is a compact group with unit $e$ and if $X$ is a compact space with multiplication $x y=y$ for all $x, y \in X$, then $S=G \times X$ is a compact mob, using coordinate-wise multiplication. We have $E=E(S)=\{e\} \times X, P(S)=S$, and each maximal subgroup of $S$ is $G \times\{x\}, x \in X$.

It is a corollary to Theorem 2.5 (Gelbaum-Kalisch-Olmsted, Iwasawa, Peck) that a compact mob with two-sided cancellation is a topological group.

The results in this section do not exhaust our knowledge of the structure of $S$ related to its maximal subgroups. We cite without further comment the papers of Faucett [8], Numakura [21], Koch $[15 ; 16]$ and Koch-Wallace [17]. The maximal subgroups will continue to appear as we proceed.

3. Submobs. We define, for $x \in S$,

$$
\begin{aligned}
\Gamma_{n}(x) & =\left\{x^{n}, x^{n+1}, \cdots\right\}^{*}, \\
\Gamma(x) & =\Gamma_{1}(x), \\
K(x) & =\cap\left\{\Gamma_{n}(x) \mid n \geqq 1\right\} .
\end{aligned}
$$

Because of its elegance, simplicity, and useful character we state first a result which can be materially strengthened (Koch [15], Numakura [21], Peck [23]).

THEOREM 3.1. If $\Gamma(x)$ is compact, then $K(x)$ is the minimal ideal of $\Gamma(x)$ as well as the unique maximal subgroup of $\Gamma(x)$. If $O(x)$ $=\left\{x, x^{2}, \cdots\right\}$ then 


$$
\Gamma(x)=K(x) \cup\{O(x) \backslash K(x)\}
$$

and $O(x) \backslash K(x)$ is a discrete open subset of $\Gamma(x)$.

The first person to examine the structure of $\Gamma(x)$ seems to have been Koch [15] from whose results we quote the following.

TheOREM 3.2. (a) If $\Gamma(x)$ is locally compact and is either zerodimensional with unit or an algebraic group, then $\Gamma(x)$ is a compact topological group.

(b) If $\Gamma(x)$ is compact, then any one of the following is sufficient to imply that $\Gamma(x)$ is a group:

(i) $\Gamma(x)$ has a unit.

(ii) $\Gamma(x)$ is connected.

(iii) $\Gamma(x)=\Gamma(a)$ for some $a \neq x$.

(iv) $\{x\}$ is not open in $\Gamma(x)$.

Koch [16] has been able to generalize Theorem 3.1 in an interesting fashion but lack of space does not allow inclusion of this result.

Of capital importance in group-theory is the device of "translating" to the identity. This is not possible in mobs and an ingenious substitute for this is due independently to Koch [15] and Numakura [21].

THEOREM 3.3. If $\Gamma(x)$ is compact and if $A$ is a compact set such that $x A \subset A$, then

$$
e A=\bigcap\left\{x^{n} A \mid n \geqq 1\right\}
$$

where $e$ is the unit of $K(x)$.

We can "dualize" this result as follows (see [34]).

Theorem 3.4. Let $\Gamma(x)$ and $A$ be compact. If $A \subset x A$, then $y A=A$ for each $y \in \Gamma(x)$ and each such $y$ acts as a homeomorphism on $A$.

Both of these results have two-sided analogues (see [34]) and an interesting interpretation connecting them with a result due to $G$. T. Whyburn [37]. They also have extensions to the case of a mob acting on a space, [17] and [34].

It is a corollary to Theorem 3.1 that if $S$ is compact then $E \neq \square$. This result has been used by Wendel [36] to show Haar measure exists on a compact group.

THEOREM 3.5. Let $S$ be compact, let $J$ be a nonvoid family of nonvoid sets of $S$ and let $R=\bigcup\{T \mid T \in J\}$. If

(i) $T_{1}, T_{2} \in J$ implies $T_{3} \subset T_{1} \cap T_{2}$ for some $T_{3} \in J$ and if

(ii) $t \in R$ and $T \in J$ imply $T_{1} \subset t T$ and $T_{2} \subset T t$ for some $T_{1}, T_{2} \in J$, 
then

$$
S_{0}=\cap\left\{T^{*} \mid T \in J\right\}
$$

is a group and the minimal ideal of the smallest closed submob of $S$ containing $R$.

This result, a manifest extension of (3.1), is a generalization of a theorem of Peck's [23]. It has several reformulations which the reader may find for himself or in [34].

If $S$ is a mob and if $G$ is an algebraic subgroup of $S$ when is $G$ a topological group, i.e., under what conditions on $G$ or $S$ can we assert that inversion is continuous in $G$ ? The first important result of this kind stems from Montgomery and asserts that if $G$ is separable metric and complete then $G$ is topological [19]. Other versions have been given by Ellis [7], N. J. Rothman (unpublished), and Moriya [20]. The latter's theorems can be extended as we shall see in (3.6) and (3.7).

Recall that a space is rim compact if each point has a neighborhood basis of open sets with compact boundaries. A rim compact Hausdorff space is completely regular. This follows from the fact that if $X$ is one such and if $A$ is a closed set with a compact boundary, then $A$ has a neighborhood basis of open sets with compact boundaries. We recall that a space is locally connected if each point has a neighborhood basis of connected sets.

Despite several published assertions that "a countably compact mob with two-sided cancellation is a topological group" there remains some doubt as to the validity of this claim. The techniques of McShane [41] and Pettis ([42], [43] and [44]) may be useful in this connection as well as in the problem just raised concerning the continuity of inversion. The results of Rothman were obtained, in part, in this framework.

TheOREm 3.6. If $G$ is a maximal subgroup of $S$ and if $S$ is locally connected and rim compact, then $G$ is topological.

TheOREM 3.7. If $S$ is a locally compact algebraic group and if the unit-component of $S$ is compact, then $S$ is topological.

We can extend (3.7) in the manner of (3.6) but we select this special case so that we may indicate a proof. Since the unit-component $C$ of $S$ is compact there is a compact open set $V \supset C$. Let $T=\{x \mid x V \subset V\}$. It is easy to see that $T$ is an open and closed submob ([28] and [31]) and is compact because $u \in V$. Now $T$ has twosided cancellation because $S$ has this property so that (remarks fol- 
lowing (2.5)) $T$ is an open topological subgroup. Thus inversion is continuous at $u$ and, since $x \rightarrow x^{-1}$ is an antihomomorphism, its continuity at $u$ implies its continuity on $S$.

The methods in this proof are of general application. The reader will note that $V$ might have been selected inside any open set about $C$ and so as a corollary we have the familiar result that any totally disconnected locally compact group contains arbitrarily small open compact subgroups. The fact that $S$ was an algebraic group did not appear until the last two sentences so we can say that a locally compact totally disconnected mob with unit has arbitrarily small open compact submobs containing the unit. We can easily generalize this to the case where the component of $S$ containing an idempotent is compact.

The familiar process of dividing a group by its unit-component has a mob-theoretic version. Let $S$ be a locally compact mob with each component compact. If $T$ is the component space of $S$ we construct in an obvious way a continuous multiplication in $T$ and a (continuous) homomorphism $f$ of $S$ onto $T$. Of course $T$ is locally compact and totally disconnected. The map $f$ is closed but need not be open.

The use of sets like $\{x \mid x A \cap B \neq \square\}$ is typical. This device seems indicated as a replacement for the use of $B A^{-1}$ with which the above set would be identical were $S$ a group. It is interesting to carry through (as far as possible) the result from topological groups that, if $A$ is compact and if $B$ is closed, then $A B$ is closed.

4. Ideals. One easily sees that any left ideal meets any right ideal. Hence the collection of all ideals of $S$ has the finite intersection property and it follows that if $S$ is compact then $S$ has a minimal closed ideal. Now it is almost obvious that, in this case, any ideal contains a closed ideal. Thus $S$ has a minimal ideal if it is compact. Further $S$ has minimal left and right ideals, Numakura [21]. One way of characterizing minimal ideals is as follows (Koch [15]):

Theorem 4.1. Let $e \in E$. These are equivalent:

(i) Se is a minimal left ideal.

(ii) $\mathrm{SeS}$ is the minimal ideal.

(iii) eSe is a maximal subgroup.

Numakura [22] initiated the investigation of nil-elements in a mob with zero and some extensions and completions have been given by Koch [16]. One can say that $x \in S$ ( $S$ with zero) is nil if $0 \in \Gamma(x)$. It is possible to extend Theorem 4.1 in various ways using the notion of nil-elements. Despite the interest and importance of this material we are impelled by its lack of definitive status to go no further in this 
direction. A purely algebraic situation has been considered by Clifford, Rich, and Schwarz (see bibliography in [4]).

Maximal ideals were first investigated by Koch and Wallace [17]. They are of great importance both structurally and as a tool. If $A \subset S$ let $L(A)$ be the union of all left ideals of $S$ contained in $A$. Of course $L(A)$ may be null. The primary weapon here is the fact that if $A$ is closed then $L(A)$ is closed and if $A$ is open and if $S$ is compact then $L(A)$ is open. One has (see [11] and [17])

THEOREM 4.2. If $S$ is compact and if $S$ properly contains an ideal, then $S$ has a maximal proper ideal $J$. If $S$ has a unit then $J=S \backslash H(u)$.

There are many variations on this theme and many applications of the concepts involved and we shall illustrate with some typical theorems.

THEOREM 4.3. If $S$ is compact, if $S^{2}=S$, and if $E$ has at most one element, then $S$ is a group.

We note that if $S$ is connected then $J$ of Theorem 4.2 is dense in $S$ and hence we have ([17] and [31])

THEOREM 4.4. If $S$ is a clan, then $S \backslash A$ is connected for each $A \subset H(u)$.

Let us agree that a continuum is a compact connected Hausdorff space. A notable result of R. L. Moore's (see [31] for a generalization) is that a nondegenerate continuum has at least two non-cutpoints. Hence a compact connected group has no cutpoint. Theorem 4.4 extends this asserting that, if $S$ is a clan, then no point of $H(u)$ cuts $S$.

In order to show a typical way of using ideals we shall prove that if a clan is indecomposable then it is a group (see $\$ 1,[17]$, and [33]). We suppose that $S \neq H(u)$ and it follows that $K \cap H(u)=\square$. Let $U$ be an open set about $u$ with $U^{*} \cap K=\square$ and let $J$ be the union of all ideals of $S$ contained in $S \backslash U^{*}$. Thus $J$ is open and connected [17] and $J^{*}$ is a continuum not all of $S$. There are two cases. If $S \backslash J^{*}$ is connected, then $S=J^{*} \cup\left(S \backslash J^{*}\right)^{*}$ so that $S$ is the union of two of its proper subcontinua. If $S \backslash J^{*}$ is not connected then $S \backslash J^{*}$ $=A \cup B$ where $A$ and $B$ disjoint, open, and nonvoid. By a familiar result we know that $A \cup J^{*}$ and $B \cup J^{*}$ are closed connected subsets of $S$ so that again $S$ is decomposable.

In the study of continua one is led to consider $C$-sets. A $C$-set is such a subset $C$ of $S$ that if $A$ is a subcontinuum meeting $C$ then $C \subset A$ or $A \subset C$. The composants of an indecomposable continuum are $C$-sets. From [45] we have the 
Theorem 4.5. Let $S$ be a clan and let $C$ be a $C$-set.

(i) If $C \cap K \neq \square$ then $C \subset K$. If $C=K$ then $K$ is a group.

(ii) If $C \cap H(u) \neq \square$ then $C \subset H(u)$. If $e \in E$ and if $H(e)$ is nondegenerate then $C \cap H(e) \neq \square$ implies $C \subset H(e)$.

With the aid of some results which involve a type of set related to the $C$-sets one can prove, for example, the following.

TheOREM 4.6. Let $S$ be a clan and let $H(u)$ be totally disconnected. Then $S$ is semi locally connected [37] at each point of $H(u)$ and may possibly not enjoy this property at no point not in $H(u)$.

It may be seen by an example that, if $H(u)$ is connected, then $S$ may be semi locally connected at no point.

A sequence of results, combining the structure of continua with clans, has been obtained by W. M. Faucett [8]. Many of these involve the use of maximal ideals. Here are some typical ones.

THEOREM 4.7. Let $S$ be a continuum, let $q \in S \backslash K$, and let $Q$ be the component of $S \backslash\{q\}$ containing $K$. Then $q S, S q$, and $S q S$ are all contained in $Q \cup\{q\}$.

Now $q S$ is an algebraic object and $Q \cup\{q\}$ is a topological one so that the inclusion $q S \subset Q \cup\{q\}$ links these two types of things.

It follows that if $q^{2}=q$ and if $L$ is the union of all left ideals contained in $S \backslash\{q\}$, then $(S \backslash Q) q \subset L^{*} \backslash L$. If also $Q$ is a left ideal then $(S \backslash Q) q=\{q\}$.

Theorem 4.8. Let $S$ be a locally connected metric continuum and let $J$ be a maximal proper ideal of $S$. If $S \backslash J$ contains at least two points, then some arc in $S$ has only its end points in $S \backslash J$.

Finally Faucett has proved

THEOREM 4.9. Let $S$ be a clan which is irreducibly connected between two elements of $E$. Then $S$ is abelian if and only if it has a zero element.

It ought to be noted that we have presented simplified versions of Faucett's results in the interest of clarity.

We close this section with an example in which we apply some of the above results. Let $S$ be the subset of $R^{2}$ consisting of those points $(x, y)$ with $y=\sin \left(x^{-1}\right), x \geqq 1$, together with the segment from $(0,1)$ to $(0,-1)$. Note that $C$ is a $C$-set of $S$. We show that $S$ cannot support the structure of a mob with unit. Suppose this is false. By (4.4) we know that $u \in C$ or $u$ is the end point of $S$. If $u \in C$ then $C \subset H(u)$ by (4.5) (ii). If $D$ is the component of $H(u)$ containing $C$, then $D$ is a group and hence homogeneous. But clearly no closed connected sub- 
set of $S$ containing $C$ can be homogeneous. Hence $u$ is the end point of $S$. We may write $S \backslash C=\bigcup\left\{A_{i} \mid i=1,2, \cdots\right\}$ where $A_{1} \subset A_{2}$ $C A_{3} \cdots$ and $A_{i}$ is an arc with $u$ as one end point. For each $i$ we know that $A_{i} C$ is a locally connected continuum containing $C$ and thus $A_{i} C=C$. Thus $C=(S \backslash C) C$ and $C^{*}=(S \backslash C)^{*} C=S C$. Similarly $C=C S$. Thus $C$ is an ideal and hence $K \subset C$. By (4.5) (i) $C \subset K$ and $K$ is a group. But $C$ is not homogeneous.

5. Algebraic generalities. The definitions which we now give are to be used in the next section. They have, it may seem, no a priori intuitive raison d'etre.

Let $X$ be a space and let $G$ be some discrete additive abelian group. If $n \geqq 0$ let $\phi$ and $\psi$ be any two functions on $X^{n+1}$ into $G$. We define an addition by

$$
(\phi+\psi)\left(x_{0}, x_{1}, \cdots, x_{n}\right)=\phi\left(x_{0}, x_{1}, \cdots, x_{n}\right)+\psi\left(x_{0}, x_{1}, \cdots, x_{n}\right) .
$$

We let $0\left(x_{0}, x_{1}, \cdots, x_{n}\right)=0 \in G$ and $(-\phi)\left(x_{0}, x_{1}, \cdots, x_{n}\right)$ $=-\phi\left(x_{0}, x_{1}, \cdots, x_{n}\right)$. With these operations the set $C^{n}(X, G)$ of all functions on $X^{n+1}$ to $G$ becomes a group. A function $\phi \in C^{n}(X, G)$ is locally zero if, for each $x \in X$, there is an open set $U$ about $x$ such that $\phi\left(x_{0}, x_{1}, \cdots, x_{n}\right)=0$ if $x_{0}, x_{1}, \cdots, x_{n} \in U$. If $\phi \in C^{n}(X, G)$ we define a function $\phi^{\prime} \in C^{n+1}(X, G)$ by

$$
\phi^{\prime}\left(x_{0}, x_{1}, \cdots, x_{n+1}\right)=\sum_{i=0}^{n+1}(-1)^{i} \phi\left(x_{0}, \cdots, x_{i-1}, x_{i+1}, \cdots, x_{n+1}\right)
$$

where $(-1)^{n} g=g$ if $n$ is even and $(-1)^{n} g=-g$ if $n$ is odd, $g$ being any element of $G$. The set of all $\phi \in C^{n}(X, G)$ such that $\phi^{\prime}$ is locally zero is denoted by $Z^{n}(X, G)$. A simple argument shows that $Z^{n}(X, G)$ is a subgroup of $C^{n}(X, G)$. We define next a subgroup $B^{n}(X, G)$ of $Z^{n}(X, G)$ by taking $B^{0}(X, G)=0$ and by stipulating that $\phi \in B^{n}(X, G)$ $(n>0)$ if $\phi-\psi^{\prime}$ is locally zero for some $\psi \in C^{n-1}(X, G)$. Here we need the fact that $\left(\psi^{\prime}\right)^{\prime}=0$ and the fact that, since $\phi-\psi^{\prime}$ is locally zero, $\left(\phi-\psi^{\prime}\right)^{\prime}$ is also locally zero. Finally we let

$$
H^{n}(X, G)=Z^{n}(X, G) / B^{n}(X, G) .
$$

The group $H^{n}(X, G)$ is the $n$-dimensional Alexander-Kolmogoroff cohomology group of $X$ with coefficients in $G$. Modulo some easily verified assertions the reader has had a brief and painless (if unmotivated) construction of a cohomology group. We refer to Spanier [26], who gave the first exposition of this theory, for details. Further results will be found in $[26 ; 28 ; 29$ and 30$]$.

We shall now sketch some salient items of the $A K$ theory to be 
used in the next section. We sometimes conceal the coefficient group $G$ so that $H^{n}(X, G)$ becomes $H^{n}(X)$. If $f: X \rightarrow Y$ is a map then one defines a homomorphism $f^{*}: H^{n}(Y) \rightarrow H^{n}(X)$ roughly as follows: If $\phi \in Z^{n}(Y)$ let $\left(f^{\#} \phi\right)\left(x_{0}, x_{1}, \cdots, x_{n}\right)=\phi\left(f\left(x_{0}\right), f\left(x_{1}\right), \cdots, f\left(x_{n}\right)\right)$ and pass to the quotient group. In particular if $A \subset X$ and if $i: A \subset X$ is defined by $i(x)=x$, then $i^{*}: H^{n}(X) \rightarrow H^{n}(A)$ is called the natural homomorphism. While the elements of $H^{n}(X)$ are not functions (though those of $Z^{n}(X)$ are functions) it is useful to employ a function-like notation. If $h \in H^{n}(X)$ and if $A \subset X$ we let $h \mid A$ be the image of $h$ under the natural homomorphism.

If $X$ is compact Hausdorff and if $h \in H^{n}(X)$ is not zero, then there is at least one closed set $A \subset X$ such that $h \mid A \neq 0$ but $h \mid A_{0}=0$ if $A_{0}=A_{0}{ }^{*} \subset A$ and $A_{0} \neq A$. We call $A$ a floor for $h$ (see [28] and [29]). If $A=A^{*} \subset X$ and if $h \in H^{n}(A)$ is such that for no $h_{0} \in H^{n}(X)$ do we have $h_{0} \mid A=h$, then for at least one closed set $R \subset X$ we know that:

(i) If $h_{0} \in H^{n}(R \cup A)$ then $h_{0} \mid A \neq h$, and

(ii) if $R_{0}=R_{0}^{*} \subset R$ and if $R_{0} \neq R$ then for some $h_{0} \in H^{n}\left(R_{0} \cup A\right)$ we have $h_{0} \mid A=h$. We call $R$ a roof for $h$ (see [28] and [29]). We may say that $h \in H^{n}(A)$ is extendable to $H^{n}(X)$ (or to $X$ ) if $h$ is the image of some element of $H^{n}(X)$ under the natural homomorphism $H^{n}(X) \rightarrow H^{n}(A)$.

6. Cohomology in clans. Earlier we raised the question as to what properties of $S$ were inherited by $K$, the minimal ideal of $S$. If $S$ is a clan then the natural homomorphism of $H^{n}(S)$ into $H^{n}(K)$ is an isomorphism onto for any $n$. This is a consequence of the more general

THEOREM 6.1. If $S$ is a continuum with left unit and if $L$ is a closed left ideal of $S$ such that $t_{0} S \subset L$ for some $t_{0} \in S$, then the natural homomorphism of $H^{n}(S)$ into $H^{n}(L)$ is an isomorphism onto [35].

Of course in the above we have left out "for each $n$ and each coefficient group."

We obtain from this the

Corollary. If $S$ is a clan and if $e \in E$, then

$$
H^{n}(S) \approx H^{n}(e S) \approx H^{n}(e S e) .
$$

Now suppose that $n>0$, that $h \in H^{n}(S)$, and that $S$ is a floor for $h$. If $e \in E \cap K$ and if $e S e \neq S$ then $h \mid e S e=0$, contrary to the isomorphism of the corollary. So $e S e=S$ and $S$ is a group. Now a manifold $M$ has the property that for some $n>0$ and some coefficient group $G$ there is an $h \in H^{n}(M, G)$ with $M$ a floor for $h$. Thus if a clan is locally Euclidean it is a group, as we stated earlier. 
There are some curious applications of (6.1). Let us say that a topological lattice is a pair of maps, $\vee: X \times X \rightarrow X$ and $\wedge: X \times X \rightarrow X$, satisfying the usual conditions. Let us suppose that $X$ is compact Hausdorff. We readily verify that the intersection of the family of sets $\{a \vee X \mid a \in X\}$ is a single element, a unit for $X$. Dually $X$ has a zero. If we let $C$ be any component of $X$ then $C$ is also a topological lattice and hence $C$ is a clan and so $H^{n}(C)=0$ for $n>0$. It follows easily that $H^{n}(X)=0$ for $n>0$. Thus all compact topological lattices are cohomologically trivial. With the aid of some earlier results we can also prove the

Alphabet Theorem. If the letters of the alphabet are written in block capitals, then only those letters homeomorphic with I can be topological lattices.

With the aid of (6.1) one can exhibit finite-dimensional homogeneous continua that are not clans. It is an open question whether or not a finite-dimensional clan which is also a homogeneous space is a group.

Following Haskell Cohen we define the codimension of the compact Hausdorff space $X$ (relative to $G$ ) by $\operatorname{cd}(X, G) \leqq n$ if $H^{n}(X, G)$ $\rightarrow H^{n}(A, G)$ is onto for all closed $A \subset X$. Cohen [6] has justified this definition by proving the expected theorems and relations.

It is perhaps reasonable to think that if $N$ is a "large" subgroup of $S$ then the structure of $N$ ought to approximate that of $S$. For example, let $S$ be a clan and let $V$ be a nonvoid open set contained in $H(u)$. It may be seen that the translates of $V$ by elements of $H(u)$ must fill $H(u)$. Hence $H(u)$ is both open and closed and thus $H(u)=S$ because $S$ is connected. Thus if $H(u)$ contains a non-null open set then the approximation of $H(u)$ to $S$ leaves nothing to be desired! The next result leads to a proof that, under suitable conditions, the approximation is good but need not be devastatingly so. In Theorem 6.2 and its corollaries it is assumed that $S$ is a continuum and that $\operatorname{cd}(S, G) \leqq n$ (see [35] and Topological invariance of ideals in mobs, Proc. Amer. Math. Soc. vol. 5 (1954) pp. 866-868).

Theorem 6.2. Let $A$ be closed in $S$, let $p, q \in S$, and define $f: A \rightarrow q A$ by $f(x)=q x$. If $h \in H^{n}(q A, G)$ and if $h \mid(p A \cap q A)=0$, then $f^{*}(h)=0$. If also $q^{2}=q$ and $q A \subset A$ then $h=0$.

Corollary 1. Let $N$ be a closed set in $S$ with $H^{n}(N, G)=0$ and with $N \subset t_{1} N$ for some $t_{1} \in S$. Then $K$, the minimal ideal of $S$, is also a minimal right ideal and $K$ contains every floor of every nonzero $h \in H^{n}(N, G)$ and each such floor is a left ideal for $S$. If also $N \subset t_{2} N$ for some $t_{2} \in S$, then $K$ is a group and is the unique floor for each nonzero $h \in H^{n}(N, G)$. 
CoRollary 2. Let also $S$ have a unit and let $N$ be a closed subgroup of $S$ with $H^{n}(N, G)=0$. Then $K=N, K$ is a homomorphic retract of $S$ (Clifford-Miller [5]) and $H^{p}\left(S, G_{0}\right) \approx H^{p}\left(N, G_{0}\right)$ for any $p \geqq 0$ and. any $G_{0}$.

ZuSATZ ZUM zuSATZ. Let $S$ and $T$ be clans and let $f: S \rightarrow T$ be $a$ homeomorphism onto. If $\operatorname{cd}(S, G) \leqq n$ and if $H^{n}(S, G) \neq 0$, then $f$ maps the minimal ideal of $S$ onto the minimal ideal of $T$.

It is easy to see by an example that $f$ need not be homomorphic.

In the space $Z \times R$ of one complex and one real coordinate (using coordinate-wise multiplication) let $C$ be the set of all $(z, t)$ with $|z|=1$ and $t=0$ and let $W$ be the set of all $(z, t)$ with $Z=\exp (2 \pi i a)$, $t=\exp (-a), a \geqq 0$. One easily shows that $C \cup W$ is a clan. Now with the same space and any multiplication suppose that $S=C \cup W$ is a clan. We shall show that $C$ is the minimal ideal of $S$ and that $u$ is the end point of $S$. We apply Corollary 1 and its "left-right dual" and infer from $H^{1}(C, G) \neq 0$ (any $G \neq 0$ ), that $C=K$. If $u \in K$ then $S=H(u)$. But clearly $S$ cannot be a group. By Theorem 4.4 we see that $u$ is the end point of $S$.

An immediate result of Corollary 2 is that if $S$ is a clan, if $\operatorname{cd}(S, G) \leqq n$, and if $H^{n}(H(u), G) \neq 0$, then $S$ is a group. A more rapid and direct proof of this is as follows. In the exact sequence of the triple $(S, H(u) \cup K, K)$ we have $H^{n}(S, K)=0$ by $(6.1)$ and $H^{n+1}(S, H(u) \cup K)=0$ since cd $S \leqq n$. Hence $H^{n}(H(u) \cup K, K)=0$. If $S$ is not a group then $K \cap H(u)=\square$ by (2.5). By excision $H^{n}(H(u)$ $\cup K, K) \approx H^{n}(H(u))$.

An inspection of examples lends credence to the idea that, if $S$ is a clan, then in some intuitive sense $H(u)$ is contained in the "boundary" of $S$. In certain cases we can make this exact [34].

THEOREM 6.3. If $S$ is a clan topologically contained in $R^{n}(n \geqq 2)$, then $H(u) \subset F(S)$, the boundary of $S$ relative to $R^{n}$.

THEOREM 6.4. If $S$ is a clan topologically contained in $R^{n}(n \geqq 2)$ and if $I$ is a closed ideal containing $F(S)$, then $I=S$.

In the remainder of this section let $S$ be a clan.

Let us say that $A \subset S$ is covariant if $A$ is closed and if $H(u) A H(u)$ $\subset A$. It is not hard to see that, if $f(A) \subset A$ for each homeomorphism $f$ of $S$ onto $S$, then $A$ is covariant if $A=A^{*}$. If $A$ is covariant then $S A S \cap H(u)=A \cap H(u)$ and hence $A \cap H(u) \neq \square$ implies $H(u) \subset A$. On the other hand if $I$ is an ideal containing $A$ then $A \subset S A S \subset I$. Thus, when $A$ is covariant the following are equivalent: " $H(u) \subset A$," " $H(u) \cap A \neq \square$ " and "No proper ideal of $S$ contains $A$." So (6.3) and 
(6.4) are the same, because of Brouwer's theorem on regional invariance, and either follows if we can show that $H(u) \cap F(S) \neq \square$. We shall now show how it is possible to make an extension. For this purpose we need some lemmas.

Lemma 1 [28]. Let $X$ be fully normal and let $A$ be closed. If $R$ is a roof for $h \in H^{n}(A), n>0$, then $R$ is a roof for $h \mid R \cap A,(R \backslash A)^{*}=R$, and $R \backslash A$ is connected.

For a fixed $n>0$ and a fixed coefficient group $G$ we shall say that $x \in F^{n}(X)$ provided that if $U$ is an open set about $x$ then there is an open set $V$ about $x$ with $V \subset U$ such that the natural homomorphism of $H^{n}(X)$ into $H^{n}(X \backslash V)$ is onto. If $X$ is a closed set in $R^{n+1}$, then $F(X)=F^{n}(X)$ where $F(X)$ is the boundary of $X$ in $R^{n+1}$, see [28]. Further we say that $X$ is of type $F^{n}$ (of course relative to the obscured coefficient group) if for each closed $A \subset X$ we have $F(A) \subset F^{n}(A)$, where $F$ means boundary in $X$.

Lemma 2 [28]. Let $X$ be fully normal, let $A$ be closed, and let $R$ be a roof for some element of $H^{n}(A), n>0$. If $F(R) \subset F^{n}(R)$, then $R \backslash A$ is open and $F(R \backslash A)=R \cap A$. Hence if $R \cup A \neq X$, then $A$ cuts $X$ into the sets $X \backslash(R \cup A)$ and $R \backslash A$ and the latter is connected.

A slightly different form of this result was found by J. W. Keesee [Proc. Amer. Math. Soc. vol. 5 (1954) p. 193].

Lemma 3. Let $X$ be a compact Hausdorff space of type $F^{n}$ and let $A$ be a closed set with null interior which does not cut $X$. If some $h \in H^{n}(A)$ is not extendable to $H^{n}(X)$, then $X$ is a roof for $h$ and $F^{n}(X) \subset A$.

Proof. Let $R$ be a roof for $h$. If $R \cup A \neq X$ then $A$ cuts $X$, by Lemma 2. Since this is impossible, $R \cup A=X$. By Lemma $1, R=X$ since $A^{0}=\square$. If $x \in F^{n}(X)$ and if $x \in X \backslash A$, then, for some open set $V$ about $x$ not meeting $A$, the natural homomorphism $H^{n}(X)$ $\rightarrow H^{n}(X \backslash V)$ is onto. Since $X$ is a roof for $h$ we know that $h$ is extendable to $H^{n}(X \backslash V)$ and hence to $H^{n}(X)$, a contradiction.

TheOREM 6.5. Let $S$ be of type $F^{n}$ and let some $h \in H^{n}(H(u))$ be not extendable to $H^{n}(S)$, e.g., $H^{n}(S)=0$ and $H^{n}(H(u)) \neq 0$. Then $F^{n}(S)$ $\subset H(u)$ and if $F^{n}(S) \neq \square$ then $F^{n}(S)=H(u)$.

From Lemma 3 we see that $F^{n}(S) \subset H(u)$. Thus, if $F^{n}(S) \neq \square$, then $H(u) \subset F^{n}(S)$ since $F^{n}(S)$ is clearly covariant.

An examination of set-theoretic analogs of such theorems as (6.5) will tend to throw some light on this situation. If $S$ is irreducible (as a continuum) about the closed set $A$ then $A \subset S A S$ implies $S A S=S$ 
because $S A S$ is a continuum. Hence, in virtue of the earlier remarks, $H(u) \subset A$ if $A$ is also covariant. Now $S$ is irreducible (as a continuum) about the closure, $A$, of the set of its non-cutpoints and since $A$ is covariant, then $H(u) \subset A$. The set $A$ is a sort of "boundary" for $S$.

On the basis of (6.5) and an unpublished result of R. H. Bing it can be shown that if $S \subset R^{n}, n \geqq 2$, and if $H(u)=F(S)$ then $H(u)$ is a Lie group and $H(u)$ irreducibly cuts $R^{n}$ into the two connected sets $S \backslash H(u)$ and $R^{n} \backslash S$.

7. Conclusion. It is likely that we have said those things which we ought not to have said and we have left unsaid those things which we ought to have said. In the latter category lie the algebraic results of Clifford, Dubreil, Green, Miller, Rees and Schwarz and Suschkewitsch. The papers of the Russian mathematicians are not available to me. Many results have been announced by French students of the subject. The reader may consult Mathematical Reviews.

I am greatly obliged to Professor D. D. Miller for his kindness in communicating the results of some theses prepared under his direction. The notion of a maximal subgroup and a maximal ideal appear in the elegant 1950 thesis of Helen B. Grimble.

The work of R. E. Allan, on quotient mobs, which will form a part of his dissertation, is not yet in a sufficiently complete state for exposition.

Aside from those to whose results formal recognition has been given, I record here with gratitude both the indirect and immediate aid of various members of the Tulane Topology Seminar and members of the faculty.

Finally, it is my great pleasure to acknowledge the support of this work, insofar as it involves the Tulane group, by the Office of Naval Research.

\section{REFERENCES}

1. A. H. Clifford, $A$ system arising from a weakened set of group postulates, Ann. of Math. vol. 34 (1933) pp. 865-871.

2. - Semigroups admitting relative inverses, Ann. of Math. vol. 42 (1941) pp. 1037-1049.

3. - Semigroups containing minimal ideals, Amer. J. Math. vol. 70 (1948) pp. 521-526.

4. - Semigroups without nilpotent ideals, Amer. J. Math. vol. 71 (1949) pp. $834-844$.

5. A. H. Clifford and D. D. Miller, Semigroups having zeroid elements, Amer. J. Math. vol. 70 (1948) pp. 117-125.

6. Haskell Cohen, $A$ cohomological definition of dimension for locally compact Hausdorff spaces, Duke Math. J. vol. 21 (1954) pp. 209-224.

7. Robert Ellis, Continuity and homeomorphism groups, Proc. Amer. Math. Soc. vol. 4 (1953) pp. 969-973. 
8. W. M. Faucett, Topological semigroups and continua, Tulane University Dissertation, 1954.

9. L. Fuchs, On semigroups admitting relative inverses, Publ. Math. Debrecen vol. 1 (1950) pp. 227-231.

10. B. Gelbaum, G. K. Kalisch and J. M. H. Olmsted, On the imbedding of topological semigroups and integral domains, Proc. Amer. Math. Soc. vol. 2 (1951) pp. 807821.

11. A. D. Wallace, Struct ideals, Proc. Amer. Math. Soc. (in press).

12. J. A. Green, On the structure of semigroups, Ann. of Math. vol. 54 (1951) pp. 163-172.

13. Einar Hille, Functional analysis and semi-groups, Amer. Math. Soc. Colloquium Publications, vol. 31, 1948. 249.

14. J. W. Keesee, On the homotopy axiom, Ann. of Math. vol. 54 (1951) pp. 247-

15. R. J. Koch, On topological semigroups, Tulane University Dissertation, 1953.

16. - Remarks on primitive idempotents in compact semigroups with zero, Bull. Amer. Math. Soc. vol. 5 (1954) pp. 828-833.

17. R. J. Koch and A. D. Wallace, Maximal ideals in topological semigroups, Duke Math. J. vol. 21 (1954) pp. 681-685.

18. H. B. Mann, On certain systems which are almost groups, Bull. Amer. Math. Soc. vol. 50 (1944) pp. 879-888.

19. Deane Montgomery, Continuity in topological groups, Bull. Amer. Math. Soc. vol. 42 (1936) pp. 879-882.

20. Mikaa Moriya, Theorie der Halb-topologischen Gruppen und Körper, Math. J. Okayama Univ. vol. 1 (1952) pp. 109-124.

21. K. Numakura, On bicompact semigroups with zero, Bull. Yamagata Univ. (Natural Science) vol. 4 (1951) pp. 405-412.

22. - On bicompact semigroups, Math. J. Okayama Univ. vol. 1 (1952) pp. 99-108.

23. J. E. L. Peck, An ergodic theorem for a noncommutative semigroup of linear operations, Proc. Amer. Math. Soc. vol. 2 (1951) pp. 414-421.

24. D. Rees, On semi-groups, Proc. Cambridge Philos. Soc. vol. 36 (1940) pp. 387400.

25. Hans Samelson, Topology of Lie groups, Bull. Amer. Math. Soc. vol. 58 (1952) pp. 2-37.

26. E. H. Spanier, Cohomology theory for general spaces, Ann. of Math. vol. 49 (1948) pp. 407-427.

27. A. Suschkewitsch, Über die endlichen Gruppen, Math. Ann. vol. 99 (1928) pp. $30-50$.

28. A. D. Wallace, Outline for algebraic topology, Tulane University, 1949 and 1952.

29. —, A theorem on endpoints, Anais Acad. Brasil. Ci. vol. 22 (1950) pp. 29-33.

30. - The map excision theorem, Duke Math. J. vol. 19 (1952) pp. 177-182.

31. - - A note on mobs I, Anais Acad. Brasil. Ci. vol. 24 (1952) pp. 329-334.

32. - A note on mobs II, ibid. vol. 25 (1953) pp. 335-336.

33. - Indecomposable semi-groups, Math. J. Okayama Univ. vol. 3 (1953) pp. 1-3.

34. - Inverses in Euclidean mobs, ibid. vol. 3 (1953) pp. 23-28.

35. - Cohomology, dimension and mobs, Summa Brasiliensis Mathematicae vol. 3 (1953) pp. 43-54. 
36. J. G. Wendel, Haar measure and the semigroup of measures on a compact group, Proc. Amer. Math. Soc. vol. 5 (1954) pp. 923-929.

37. G. T. Whyburn, Analytic topology, Amer. Math. Soc. Colloquium Publications, vol. 28.

38. W. M. Faucett, Compact semigroups irreducibly connected between two idempotents, Proc. Amer. Math. Soc. (in press).

39. - Topological semigroups and continua with cutpoints, ibid. (in press).

40. W. M. Faucett and R. J. Koch, Complements of maximal ideals in semigroups (to appear).

41. E. J. McShane, Images of sets satisfying the condition of Baire, Ann. of Math. vol. 51 (1950) pp. 380-386.

42. B. J. Pettis, On continuity and openness of homomorphisms in topological groups, ibid. pp. 293-308.

43. - Uniform Cauchy points and points of equicontinuity, Amer. J. Math. vol. 73 (1951) pp. 602-614.

44. - Remarks on a theorem of E. J. McShane, Proc. Amer. Math. Soc. vol. 2 (1951) pp. 166-171.

45. A. D. Wallace, The position of $C$-sets in semigroups, ibid. (in press).

46. T. Rado and P. V. Reichelderfer, Continuous transformations in analysis, Springer (in press).

The Tulane University of Louisiana 